\title{
A REVIEW ON NANOPARTICLES IN TRANSDERMAL DRUG DELIVERY: POLYMERS AT VARIANCE WITH SEMICONDUCTORS AND LIPIDS
}

\author{
Ali Hany \\ STEM \\ Alexandria STEM School, Alexandria, Egypt
}

\begin{abstract}
Transdermal drug delivery system is a quest for the scientist in the last decades due to its advantages of taking away the harms of gastrointestinal and intravenous problems. Nanoparticles came to finalize this quest; in this paper polymer-based nanoparticles are compared with those of semiconductors and lipids.
\end{abstract}

Polymer-based nanoparticles are biodegradable made from natural polymers like gelatin and others or synthetic polymers like polycaprolactone and others. They can contain the drug inside or adsorbed on the outside. The polymer-based nanoparticles typically pass through diseased skin but they can pass through the hair follicles as well. Polymer-based nanoparticles propose the advantages of being stable controlled and give sustained drug release.

Semiconductor nanoparticles are hollow copper sulfide nanoparticles that carry the drug inside but to face the stratum corneum thermal ablation is used. The Photothermal properties of the nanoparticles allow them to absorb near-infrared light and get temperature around $40^{\circ}$ and then partially damage the stratum corneum without a touch to the skin itself allowing the drug to pass safely.

Solid lipid nanoparticles were made to deliver diclofenac sodium a potent non-steroidal anti-inflammatory drug. They are prepared through emulsion and solvent evaporation. They pass through the stratum corneum because they are made of lipids.

In the review paper, the three types of nanoparticles are at variance to discuss everything about them with a little comparison.

Keywords - Transdermal, Nanoparticles, Polymer-based, Copper sulfide, Lipid.

\section{INTRODUCTION}

The delivery of drugs across the skin was a goal for the science for decades as skin is an attractive are of the delivery due to its stability and ease of access [Alkilani, McCrudden, and Donnelly et al 2015][ Prausnitz, Mitragotri, and Langer et al 2004]. Though, due to the barrier posed by the skin, most compounds were administrated with a hypodermic needle. This has many disadvantages like the pain, needle phobia, and transmission of infections.

The most difficult challenge that stands upon the development of transdermal drug delivery is the skin barrier, stratum corneum. Stratum corneum is the first layer of skin, 15 to $20 \mu \mathrm{m}$ thick, densely packed, comprised of dead corneocytes filled with keratin, and surrounded by lipid matrix.

Nanoparticles can be known from their name they are particles in the nano dimensions that can be hollow or not and carry the drug inside or outside. In the last few decades, nanoparticles have been used in many fields of industry, medicine, and even domestic products [Anselmo, and Mitragotri, et al 2019]

[Farjadian, Ghasemi, Gohari, Roointan, Karimi, and Hamblin et al 2019]. Nevertheless, this review will show how would the nanoparticles defeat the stratum corneum and get into the body carrying the drug to help humanity. You will find how the polymers, semiconductors or lipids are all working with different strategies to solve the same challenge and where they ended up.

Polymer-based nanoparticles held the criteria of biodegradability and biocompatibility. But they managed themselves a way to get in. While semiconductor nanoparticles of hollow copper sulfide held the drugs inside and used photothermal properties. But the solid lipid nanoparticles worked on the physical properties and went through the stratum corneum holding diclofenac sodium.

The aforementioned advantages enable the nanoparticles to utilize the transdermal drug delivery system. Here, it is summarized the chemical, physicochemical and photothermal properties of nanoparticles making them crucial vehicles in nanomedicine and provide a review of the last decade for 


\section{International Journal of Engineering Applied Sciences and Technology, 2020 \\ Vol. 5, Issue 2, ISSN No. 2455-2143, Pages 27-36 \\ Published Online June 2020 in IJEAST (http://www.ijeast.com)}

improvement in the transdermal drug delivery system by the nanoparticles.

\section{BODY \& DISCUSSION}

The transdermal drug delivery system is the approach of delivery to transport the drug inside the body through the skin [Godin, Touitou et al 2009][Wiedersberg, Guy et al 2014]. It has many advantages which are taking away from gastrointestinal absorption problems and hepatic first-pass effect and etc.

According to the advantages mentioned, many research pieces were conducted to find the right way into the transdermal drug delivery system. The majority of those researches concern polymers, semiconductors, and lipids. However, the most active and the best are those regarding nanoparticles of the polymer, semiconductors, and lipids. Furthermore, they are going to be discussed, criticized, and compared.

\section{A. Polymer-Based Nanoparticles-}

The most challenging part of the transdermal drug delivery is passing the drug through the stratum corneum, which is the upper layer of the skin. So, the polymer-based nanoparticles were applied on diseased skin, where the stratum corneum is already broken.

The polymers used have to have specific properties, to achieve the intended mission, and one of the most important is to be biodegradable to be delivered safely into the body. The polymeric biomaterial material must be selected carefully since, during the degradation and the release of the drug, the decomposed products have to be non-toxic [Jeevanandam, Barhoum, Chan, Dufresne, and Danquah, et al 2018]. Many factors influence non-linear kinetic biodegradation. These include the size, shape, chain defects, ion exchange, ionic strength, $\mathrm{pH}$, morphology, microstructure, molecular weight distribution, and route of administration to the site of action and the material itself, whether natural or synthetic [Badri , Eddabra, Fessi and Elaissari et al 2014].

A.1. Natural polymers are typically biodegradable and exceptionally biocompatible. Moreover, due to the availability of different types of natural polymers nowadays, the manufacturers have gained considerable achievement regarding the development of most promising therapeutic systems that provide effective long-term therapy to the patients. There are many natural polymers, but the most important to the transdermal drug delivery are:

A.1.1. Gelatin. Gelatin is an organic polymer that is derivative of the collagen. Because gelatin is biodegradable and biocompatible, it is mainly used in the pharmaceutical and medical fields. The importance of gelatin comes from its mechanical and thermal properties and swelling comportment. Since gelatin is non-toxic, has bioactive properties, and has a reasonable price, gelatin is a new polymer for controlled release transdermal drug delivery.

A.1.2. Chitosan. Chitosan is produced through chitin's deacetylation, the structural component of cell walls of the exoskeleton of crustaceans, and fungi. It is a non-toxic polymer digested by either lysozymes enzymes or chitinases found in the blood and intestine. These properties led to enhance the interest of polymer in the area of the pharmaceutical industry and research as a vehicle for drug delivery [Haeri, Sadeghian, Rabbani, Anvari, Ghassemi, Radfar, and Dadashzadeh, et al 2017]. Furthermore, chitosan is positively charged, which consecutively permits interaction with the negatively charged mucosal surface .

A.1.3. Dextran and Its Derivatives. Dextrans are the polymers that are synthesized in bacteria from sucrose. Besides, in these polymers predominantly, the glucosidic linkages are $\alpha$ (1-6) kind. Moreover, it can be synthesized chemically as well. The excellent biocompatibility, biodegradability, and low toxicity are the main features that support the use of the polymer in the medical and pharmaceutical fields.

A.2. Synesthetic polymers are present with a variety of compositions with controllable properties. Nevertheless, the most important types of this variety of composition are:

A.2.1. Polycaprolactone (PCL). PCL is a semi-crystalline, hydrophobic, biodegradable, and biocompatible polymer. It has a slow degradation rate that makes it a favorable polymer for extended drug delivery systems.

A.2.2. Poly(lactic acid) (PLA). PLA is a biodegradable and biocompatible synthetic polyester that suffer scission in the body to monomeric units of lactic acid. It has good mechanical properties. Usually, solvent diffusion, salting out, solvent displacement salting out, and solvent evaporation are the methods which are used for the preparation of PLA nanoparticles.

A.2.3. Poly(lactic-co-glycolic acid) (PLGA). PLGA is one of the most efficaciously biodegradable polymers because its hydrolysis leads to metabolite monomers, lactic acid, and glycolic acid. They are metabolized with minimum system toxicity.

A.2.4. 'Smart' Polymers. They are called "smart" polymers because of the conformational changes that they undergo in response to changes in environmental conditions. Smart polymers can be used in different areas. They are used in intelligent systems of drug delivery. Furthermore, they release the entrapped drugs in response to physiological triggers in the suitable site of action.

After all, due to the modern nanotechnology in pharmaceutics, many advantaged has given rise from a single preparation. The diameter of the polymer-based nanoparticles would be less than $1 \mu \mathrm{m}$; they are also prepared from natural or synthetic polymers, as mentioned. Those nanoparticles can be either nanocapsules or nanospheres. Where the drug molecules are either trapped inside or adsorbed on the surface. Moreover, the drug pathway has few options for polymer-based transdermal nanoparticle delivery; the drug transport skin by passive diffusion by the paracellular, the transcellular, and the appendageal routes.

Nonetheless, biodegradable nanoparticles used to improve the therapeutic significance of different water-soluble or 


\section{International Journal of Engineering Applied Sciences and Technology, 2020 \\ Vol. 5, Issue 2, ISSN No. 2455-2143, Pages 27-36 \\ Published Online June 2020 in IJEAST (http://www.ijeast.com)}

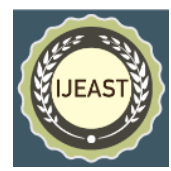

insoluble pharmaceuticals through improving solubility, retention time, and consecutively bioavailability. The achievement of ideal drug loading of carriers, drug release control, delivery, and design of stable materials that do not release dangerous degradation products challenge the design and characterization of the nanoparticles. Though, the polymerbased nanoparticles propose the advantages of being stable, controlled, and have sustained drug release. Their further advantages over lipid-based vehicles include the encapsulation of both kinds of molecules (hydrophobic and hydrophilic), storing over months, functionalization and furthermost their physicochemical properties can be adjusted, which is most important. The penetration incapability of polymeric nanoparticles to the stratum corneum is from their restriction; however, they accumulate in skin furrows and hair follicles and increase the local concentration by loaded drugs that can more diffuse to the viable layers of the skin. It should be kept in mind that for improving drug delivery to the deeper skin layers, the composition and surface charge of nanoparticles are to be considered [Badri , Eddabra, Fessi and Elaissari et al 2014].

The polymer-based nanoparticles typically interact with the stratum corneum; this layer of the skin is built from protein-rich cells packed with a lipid extracellular matrix. Moreover, corneocytes are attached through the intracellular protein structure that is called corneodesmosmes. However, polymeric particles are hydrophilic, might not have interactions with the extracellular lipids of stratum corneum. The three-dimensional conformation of stratum corneum that leads to agglomeration, destabilization, or absorption would change its interaction with polymeric carriers while they do not fuse with the lipids of stratum corneum. The healthy microbial flora of the skin surface will interact with applied materials. Besides, the sebum, which formed a shell to the stratum corneum, is rich in lipids and proteins. It can interact with administered polymer-based materials to shift their approach to interact with the other skin components or destabilize them. The $\mathrm{pH}$ of the skin surface is between 4.2 and 5.6. Degradation of the polymer-based carriers might increase by acidic $\mathrm{pH}$ of the skin. Moreover, the paracellular, the transcellular, and the trans-follicular route are the three described pathways of penetration for locally applied materials.

Nevertheless, the stratum corneum is not the only part of the skin that allows the nanoparticles to pass; the hair follicles do as well. The hair follicle canal of the pilosebaceous unit demonstrates a reservoir where micro-, submicron-, and nanosized substances can penetrate and accumulate. The size of the hair follicle orifices ranges between $102-242 \mathrm{~m}$ in terminal hair follicles and $49-123 \mathrm{~m}$ in vellus hair follicles Indeed, as demonstrated only the $0.1 \%$ of the skin surface is formed through the hair follicle orifices.

Since the last decades, the trans-follicular pathway's importance, particularly for the preferential penetration of particulate substances, has been shown in individual studies. Particles that accrue in the hair follicle are safe from stratum corneum turnover and persist for days before cleared the interaction surface of hair follicle infundibulum about 0.68 for empty follicles, and $0.068 \mathrm{~cm} 2$ for full follicles was calculated.

Consequently, the hair follicle infundibulum establishes a reservoir and a considerable surface area where interaction among accumulated substance and skin structures can occur. The hair follicle is a by-pass for a quicker penetration of substances. After the selectively closing of hair follicle orifice, the administered drug materials reached equal blood values. This could be because the stratum corneum barrier in deep infundibulum is thin, and molecules might be diffuse more promptly here than on the skin surface where the stratum corneum consists of specific more layers. Furthermore, the accumulated carriers in the hair follicle canal release the encumbered drug, creating a high local concentration of the drug that is a dynamic force for its diffusion to the viable skin. The elimination of sebum cells debris, and eventually, bacteria from the hair follicle that formed its content improved the penetration of locally administered particulate materials .

In summary, the polymer-based nanoparticles are working with main principles, biodegradability, and biocompatibility.

\section{B. Semiconductors Nanoparticles: Hollow Copper Sulfide-}

Most of the used transdermal patches used in most of the clinical products have been limited to lipophilic drugs with a molecular weight of a few hundred Daltons. This is because of the presence of lipid-rich stratum corneum barrier, percutaneous delivery of hydrophilic macromolecules such as peptides and proteins, which presents a difficult challenge. In a quest to overcome this limitation, a variety of physically enhanced transdermal technologies have been developed to interrupt the stratum corneum, such as cavitational ultrasound, electroporation, microdermabrasion, microneedles, and thermal ablation. Thermal ablation technology signified an interesting approach in recent years. This technology is principled to directly heat skin surface to generate micron-scale perforations in the stratum corneum, mainly through micro-heaters, radiofrequency, or laser. All these approaches rely on particular control over the skin disruption to diminish the injury to the surrounding and deeper tissue.

Photothermal nanoparticles are engineered particles possessing a photothermal coupling effect. For example, gold nanostructures such as nano-shells, nanorods, nanocages, and hollow nanospheres have unique optical properties due to stable and tunable surface plasmon resonance [Chen, $\mathrm{Li}, \mathrm{Zhao}, \mathrm{Wu}$, Zhu, Mao, and Gao, et al 2018]. They can be made-up to absorb near-infrared light (650-900 nm) [Feng, Zhang, Zhang, Shan, Yuan, Zhang, Hou, and Zhang et al 2016], resulting in resonance and transfer of thermal energies to the surrounding tissue to raise the temperature. The absorbance of near-infrared light is desirable because it causes minimal thermal injury to healthy tissues with ideal light penetration.

Semiconductor copper sulfide nanoparticles (CuSNPs) are a new class of photothermal nanoparticles that provide an alternative to gold analogs. Unlike the optical absorption in gold nanostructures based on the surface plasmon resonance, 
the near-infrared light absorption of semiconductor copper sulfide nanoparticles comes from the d-d transition of $\mathrm{Cu} 2+$ ions. The surface plasmon resonance peak of gold nanostructures is dependent on the dielectric constant of the surrounding medium. Therefore, the absorption wavelength of gold nanostructures will be changed by the solvent or the surrounding environment when they are formulated or delivered in vivo. In contrast, the absorption wavelength of semiconductor copper sulfide nanoparticles is not affected. Further, compared to gold, copper sulfide is much less expensive.

In these studies, a hollow copper sulfide nanoparticle (HCuSNP)-mediated transdermal delivery technique was designed. Application of nanosecond-pulsed near-infrared laser would provide brief periods but extremely high temperatures in local regions, with focused thermal ablation of the stratum corneum, limiting heat transfer to deep tissue. It is expected that the extent of thermal ablation of skin, i.e., removal of stratum corneum, viable epidermis, or dermis, would be controlled by adjusting the laser power. Moreover, the transmission electron micrograph demonstrates that the hollow copper sulfide nanoparticles are hollow and core-shell spheres with an average diameter of around $55 \mathrm{~nm}$. The nanoparticles have an optical absorption band peaking at $1050 \mathrm{~nm}$, with $\sim 80 \%$ of peak absorbance intensity at $900 \mathrm{~nm}$ of near-infrared wavelength as shown in figure 1(a) and figure 1(b) [Ramadan, Guo, Li, Yan and Lu et al 2012].

The hollow copper sulfide nanoparticles were suspended in Carbomer 940 hydrogel to be topically applied to the skin of nude mice. The role of Carbomer 940 here is to increase the viscosity of the formulation. Propylene glycol (10\%) was contained in the gel formula serving as wetting agents to increase the surface contact between the gel and the skin. After the skin was heated with visible light $20 \mathrm{~min}$, the water in the gel evaporated, and the nanoparticles were well deposited on stratum corneum. The hollow copper sulfide nanoparticles coated skin was then irradiated with neodymium:yttriumaluminum-garnet (Nd:YAG) laser in tandem with Ti:sapphire laser, whose wavelength was set at 900 $\mathrm{nm}$. Real-time infrared thermal imaging was used to monitor the skin temperature pre-, intra- and post- NIR laser irradiation. The temperature of the skin area pretreated with hollow copper sulfide nanoparticles gel elevated immediately after laser irradiation. The temperature continued to increase with laser exposure time. It reached plateaus of $40^{\circ} \mathrm{C}$ and $50^{\circ} \mathrm{C}$ after laser treatment for 5 seconds with power intensities of $1.3 \mathrm{~W} / \mathrm{cm} 2$ and $2.6 \mathrm{~W} / \mathrm{cm} 2$. Thus, the duration of laser treatment for all the experiments was set at 5 seconds. Although, the blank gel without hollow copper sulfide nanoparticles did not increase the skin temperature even at high power $(2.6 \mathrm{~W} / \mathrm{cm} 2)$.

Histology studies assessed the outcome of near-infrared laser treatment on nude mouse skin in vivo with or without hollow copper sulfide nanoparticles. Without laser treatment, the hollow copper sulfide nanoparticle gel did not bring any histological change of the skin. The blank gel-applied skin
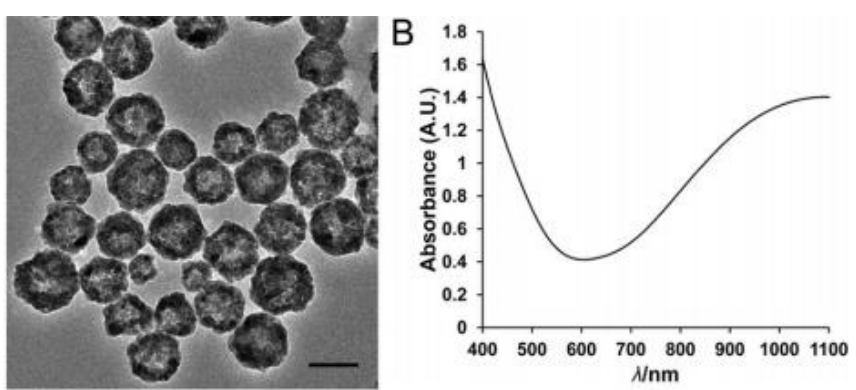

Figure1. A) Transmission electron micrograph of HCuSNPs. B) Experimental absorbance spectrum of HCuSNPs in water. Bar, $50 \mathrm{~nm}$.

remained intact after near-infrared laser irradiation at 2.6 $\mathrm{W} / \mathrm{cm} 2$. Nevertheless, significant histological changes were observed in hollow copper sulfide nanoparticle gel-applied skin following laser treatment. This damage was confined to the stratum corneum layer without damage to the viable epidermis. The pore depth was measured as $11 \pm 2 \mu \mathrm{m}$ from 10 hematoxylin $\&$ eosin (H\&E)-stained slices. Increasing the laser power to 2.6 $\mathrm{W} / \mathrm{cm} 2$ resulted in thermal ablation penetrating the viable epidermis, reaching the epidermal-dermal junction, and producing dee per pores that stretched into the dermis. The pore depth was measured as $29 \pm 7 \mu \mathrm{m}(\mathrm{n}=10)$. The application of hollow copper sulfide nanoparticle solution without gel following laser treatment showed a similar ablation effect on the skin than hollow copper sulfide nanoparticle gel, indicating the use of gel on skin surface did not disturb the energy efficiency of the near-infrared laser.

The laser-treated skin was stained with the lipid-soluble fluorescent dye, Nile red, to provide full information on the changes in stratum corneum. Mouse skin applied with hollow copper sulfide nanoparticle gel alone maintained a high degree of alignment of intercellular lipids in the stratum corneum with well-ordered stacking arrangement of corneocytes, suggesting the hollow copper sulfide nanoparticles gel itself did not substitute the structure of the stratum corneum. Furthermore, mouse skin treated with blank gel plus high-powered laser (2.6 $\mathrm{W} / \mathrm{cm} 2$ ) did not display significant changes in the stratum corneum's integrity. Though, mouse skin exposed to the hollow copper sulfide nanoparticle gel plus low powered laser treatment $(1.3 \mathrm{~W} / \mathrm{cm} 2)$ had a highly disordered stratum corneum, which was absent in certain areas. This could be attributed to the breaking of the stratum corneum keratin network. In mouse skin treated with hollow copper sulfide nanoparticle gel plus high-powered laser $(2.6 \mathrm{~W} / \mathrm{cm} 2)$, the stratum corneum was extensively injured and densely packed, with little of its original structure remaining. Some of the stratum corneum and partial epidermis structure disappeared, probably due to heat-induced chemical decomposition of the tissue. It was reported that heating the skin with short and hightemperature pulses sequentially induced a stratum corneum densely packed, disordered, and broken down due to the increase of temperature from $100^{\circ} \mathrm{C}$ to $315^{\circ} \mathrm{C}$. Moreover, 


\section{International Journal of Engineering Applied Sciences and Technology, 2020 \\ Vol. 5, Issue 2, ISSN No. 2455-2143, Pages 27-36 \\ Published Online June 2020 in IJEAST (http://www.ijeast.com)}

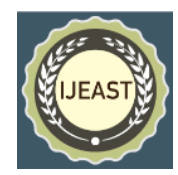

results are consistent with such deviations induced by the local heat, indicating the thermal conduction from the hollow copper sulfide nanoparticles triggered by the pulsed Nd:YAG laser to the skin could prime to temperatures exceeding $100^{\circ} \mathrm{C}$.

Compared with the continuous-wave laser power (12 $\mathrm{W} / \mathrm{cm} 2)$ previously used in copper sulfide nanoparticlemediated photothermal ablation therapy, the pulsed laser of fewer than $15 \mathrm{~ns}$ per pulse applied here $(1.3$ and $2.6 \mathrm{~W} / \mathrm{cm} 2)$ has less average power. However, the peak power of the pulsed laser dramatically exceeds that of the continuous-wave laser. Thus, the pulsed laser induces beneficial local heating by photoexcitation. Besides, the histological results proved that pulse irradiation of the hollow copper sulfide nanoparticle-applied mouse skin for only $5 \mathrm{~s}$ was sufficient to destroy the stratum corneum and even the viable epidermis locally. The previous report indicated that copper sulfide nanoparticle-mediated photothermal ablation required a 5-minute exposure to continuous-wave laser at higher power [46].

For thermal ablation-enhanced transdermal drug delivery, keeping the thermal exposure insufficiently brief periods is significant. This mode of heating enables the temperature gradient across the stratum corneum to be steep enough that the skin surface is tremendously hot but not the viable epidermis and deeper skin tissues. Therefore, "cold ablation" can be done with high energy short-duration laser pulses of microseconds or even less with limited or negligible heat transfer to nearby tissue. Alternatively, in the previously informed transdermal drug delivery system enhanced by the solid-in-oil dispersion of gold nanorods, a xenon lamp was used as a continuous near-infrared light source that essential high light power $(6 \mathrm{~W} / \mathrm{cm} 2)$ and long duration of light exposure $(20 \mathrm{~min})$ to ablate the stratum corneum, causing enormously high energy $(7200 \mathrm{~J} / \mathrm{cm} 2)$. This is a 3-order of magnitude higher than the pulsed laser energy used in our system, which was $6.5 \mathrm{~J} / \mathrm{cm} 2$ with a laser power density of $1.3 \mathrm{~W} / \mathrm{cm} 2$ for $5 \mathrm{~s}$ exposure time. Consequently, the heat propagation to deep surrounding tissue using light source from the xenon lamp could be a significant concern.

The success of the photothermal ablation improvement approach relies on the highly photothermal coupling efficiency of the hollow copper sulfide nanoparticles at the near-infrared wavelength region. Application of very short (femtosecond to nanosecond) pulsed near-infrared laser allows a large amount of energy to be tightly packed in the laser pulses, producing rapid heating in the nanoparticles. In this case, the temperature of nanoparticles could be raised by several hundred degrees. This is followed by rapid heat transfer out of the particle to the adjacent tissues and quick cooling of the nanoparticles at the end of each laser pulse. Instantaneous heat conduction could allow an increase in temperature exceeding $100^{\circ} \mathrm{C}$ to be localized. As the discontinuous light of the pulsed laser causes less heat accumulation than continuous wave laser, the average temperature of the irradiated skin area only increases to $~ 40-$ $50^{\circ} \mathrm{C}$. Nonetheless, this kind of heat conduction could cause disordering of stratum corneum lipid construction, disruption of stratum corneum keratin network structure, or decomposition and vaporization of keratin to micron-scale holes in the stratum corneum. The disrupted skin surface subsequently allows substantial absorption of macromolecules.

The current work proved that a near-infrared laser-activated transdermal drug delivery mediated by hollow copper sulfide nanoparticles is an effective large-molecule-drug delivery technique. The comparative study proved this photothermal ablation enhancement has the advantage of permeability to macromolecules over chemical enhancement. The permeability enhancement by chemical enhancers is caused by interference with the packing arrangement of the intercellular lamellar lipids in the stratum corneum through the insertion of amphiphilic molecules into the lipid bilayers, or by extracting lipids using solvents and surfactants to create lipid packing defects of nanometer dimensions. Thus, chemical enhancement is limited to small, lipophilic molecules and has a limited effect on larger or hydrophilic molecules. In contrast, the photothermal ablation approach increases skin permeability. It was reported that thermal ablation enhancement techniques made micrometer dimensions of disruptions to the stratum corneum constructions. These microscale disruptions created channels of sufficient dimensions for the passage of macromolecules, including antibodies. Consequently, it is believed that the hollow copper sulfide nanoparticle-mediated photothermal ablation enhancement technique can be used for the transdermal delivery of drugs with a molecular weight more significant than $22 \mathrm{kDa}$. The photothermal ablation-induced micro-scale disruptions are also perfect for antigen delivery of vaccine targets the potent epidermal Langerhans and dermal dendritic cells that generate a robust immune response at much lower doses than hypodermic.

\section{Lipid Nanoparticles: transdermal delivery of diclofenac sodium}

Those solid lipid nanoparticles were made for individual use, and administrating particular drugs; diclofenac sodium (DNa), a potent non-steroidal anti-inflammatory drug with pronounced analgesic properties, is used in the long-term treatment of rheumatoid arthritis, osteoarthritis, and ankylosing spondylitis. It is expansively metabolized in the liver, and due to its biological half-life, which is only $1-2 \mathrm{~h}$, the drug has to be given frequently. Gastrointestinal side effects, such as bleeding, ulceration, or perforation of the intestinal wall, are commonly seen [Baraf, Fuentealba, Greenwald, Brzezicki, O'Brien, Soffer, Polis, Bird, Kaur, Curtis and EDGE Study Group et al 2007]. Because of the short biological half-life and associated adverse effects, it is considered as an ideal candidate for controlled transdermal drug delivery.

The transdermal drug delivery system promises many advantages over oral or intravenous administration; however, the success of a transdermal drug delivery system depends on the ability of the drug to enter the skin in satisfactory quantities to maintain the therapeutic level. Various approaches have been proposed to overcome the low permeability of drugs through 


\section{International Journal of Engineering Applied Sciences and Technology, 2020 \\ Vol. 5, Issue 2, ISSN No. 2455-2143, Pages 27-36 \\ Published Online June 2020 in IJEAST (http://www.ijeast.com)}

the skin. In the past decade, solid lipid nanoparticles (SLNs) have been looked upon as one of the promising carriers for presenting several attractive features for transdermal drug delivery [Ezzati, Nazhad, Dolatabadi, Valizadeh, and Hamishehkar, et al 2015].

solid lipid nanoparticles are colloidal carrier systems for controlled drug delivery, following the development of emulsion, liposomes, microparticles, and nanoparticles based on synthetic or natural polymers. They possess numerous features that are advantageous for the topical route of application.

Most of the used lipids have been in an approved status or are excipients used in commercially available topical cosmetic or pharmaceutical preparations. Concerning their use, as carriers for topical applications, the occlusive effect was due to film formation on the skin surface that reduces trans-epidermal water loss. Increasing the water content in the skin can reduce the symptoms of atopic eczema and advance the appearance of healthy human skin. Occlusion can also improve the penetration of drugs through the stratum corneum by increased hydration. Apart from a non-specific occlusion effect on penetration, penetration might also be affected by the carriers. The small particle sizes of solid lipid nanoparticles ensure the nanoparticles being in close contact with the stratum corneum, thus increasing the number of encapsulated agents penetrating the viable skin. A controlled release of drugs from solid lipid nanoparticles could be observed owing to the solid lipid matrix. This becomes a valuable tool when it is necessary to supply the drug over a prolonged period, to reduce systemic absorption, and when drug produces irritation in high concentrations

A popular method is used for the preparation of nanoparticles, for microencapsulation of water-insoluble drugs is the solvent evaporation process. Generally, the entrapment efficiency (EE) of the water-soluble drug is low due to drug loss from the organic emulsified phase before solidification of lipid materials in the solid lipid nanoparticles. So, optimization of the process may be advantageous for the efficient entrapment of water-soluble drugs. It is envisaged to evaluate different formulation factors, including the organic phase, waterdispersed phase, co-emulsifiers, emulsifiers, and lipid matrix, to get an optimized formulate of solid lipid nanoparticles containing diclofenac sodium. In vitro cutaneous permeation studies of the solid lipid nanoparticles through rat skin were also investigated. The long-term goal of this study is to develop topical diclofenac sodium formulations for clinical use to increase the therapeutic index.

The diclofenac sodium - solid lipid nanoparticles were prepared according to a modified emulsion/solvent evaporation method. Briefly, $17.5 \mathrm{mg}$ diclofenac sodium was dissolved with the Glycerol monostearate in $5 \mathrm{~mL}$ ethanol at the temperature of $60^{\circ} \mathrm{C}$ forming the oil phase. Meanwhile, the aqueous phase was heated to the same temperature. The oil phase was added into the hot aqueous phase by drops under rapid stirring at 1200 $\mathrm{rpm}$ at $60^{\circ} \mathrm{C}$ for dispersion. Then the homogeneous suspension was poured into the dispersed phase $(25 \mathrm{~mL}$ of $1 \%$ Tween- 80 , w/v) under stirring at $1000 \mathrm{rpm}$ for $4 \mathrm{~h}$ at $2^{\circ} \mathrm{C}$ in an ice bath to allow for the hardening of the solid lipid nanoparticles. The resulting suspension was filtered through a membrane which acted to exclude the particles larger than $0.45 \mu \mathrm{m}$.

\section{C.1. Characterization of solid lipid nanoparticles.}

The solid lipid nanoparticles were characterized according to the amount of encapsulated drug, surface charge, size distribution, particle size, and morphology. Solid lipid nanoparticles were prepared by the emulsion/solvent evaporation method using the mixture of ethanol and acetone as the organic solvent, Glycerol monostearate, and phospholipid as the lipid material, and Tween-80 and Polyethylene glycol 400 as the aqueous phase and dispersed phase, respectively. The solid lipid nanoparticles produced by optimal formulation were submicrometric size (208.8 $2.0 \mathrm{~nm}, \mathrm{n} 1 / 4$ 3) with a low polydispersity index $(0.2680 .024, \mathrm{n} 1 / 43)$. The entrapment efficiency determined by HPLC by the ultracentrifuge method was about $88.82 .2 \%$ (n $1 / 43$ ), and the DL was about $9.50 .7 \%$ (n $1 / 43)$.

The transmission electron microscopy photograph revealed that the emulsion/ solvent evaporation process leads to the formation of spherical nanoparticles with a smooth surface as in figure 2 [Dongfei, Yifan, Yue, Yubing, Qing, Rui. and Qunwei et al 2010], and no apparent aggregation among the solid lipid nanoparticles was observed. By close reflection of transmission electron microscopy photographs, it was found that bright and dark images were seen, demonstrating that the combination of Glycerol monostearate and phospholipid

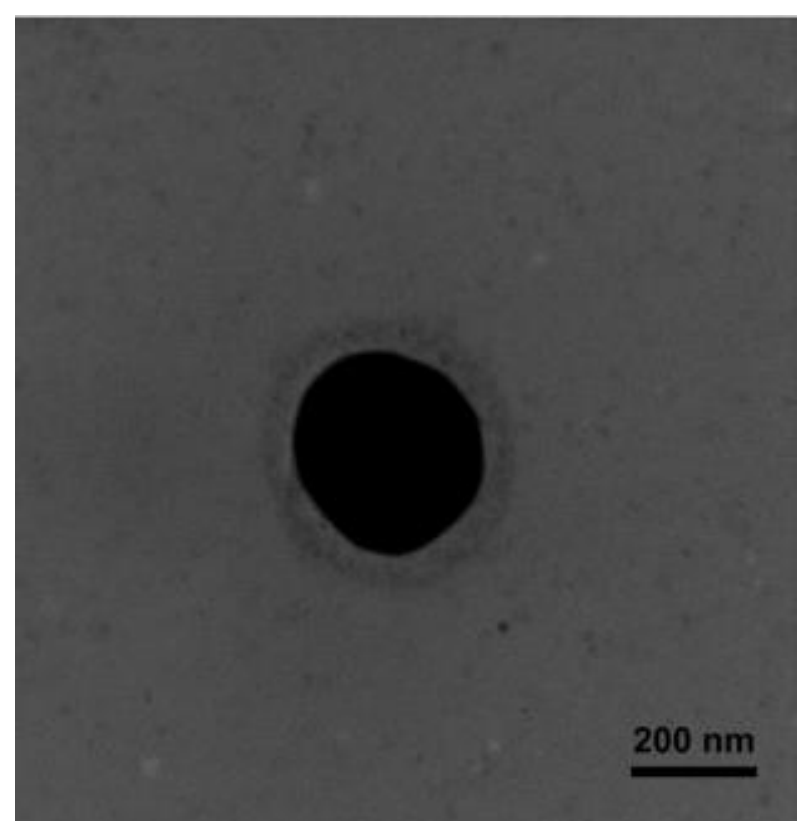

Figure 2. TEM photograph of DNa-SLNs

displayed core-shell type nanoparticles. It seemed that the dark 


\section{International Journal of Engineering Applied Sciences and Technology, 2020 \\ Vol. 5, Issue 2, ISSN No. 2455-2143, Pages 27-36 \\ Published Online June 2020 in IJEAST (http://www.ijeast.com)}

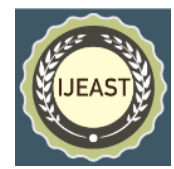

part should be assigned to the core of the Glycerol monostearate, and the bright part should be assigned to the shell of the phospholipid.

\section{C.2. In vitro release studies}

The in vitro drug release profiles of solid lipid nanoparticles in the first 720 minutes. The control profile showed that $85 \%$ of the drug was released at the first sampling time of 60 minutes and $97 \%$ by 480 minutes. The drug release from the solid lipid nanoparticles seemed to have two components with an immediate release of about $67 \%$ at the first sampling time of 120 minutes. This was followed by a slower exponential release of the remaining drug over the next 600 minutes. The fast-initial release of diclofenac sodium was possible because of the drug which was absorbed or close to the surface of the solid lipid nanoparticles and the large surface to volume ratio of the nanoparticle Geometry because of their size. Furthermore, it may also be due to the water-soluble nature of diclofenac sodium. Upon addition of the solid lipid nanoparticles to the dissolution medium, diclofenac sodium partitioned rapidly into the release medium, accounting for the burst release observed. The exponential delayed-release may be attributed to the diffusion of the dissolved drug within the lipid core of the solid lipid nanoparticles into the dissolution medium

\section{C.3. In vitro cutaneous permeation studies}

The in vitro permeation of diclofenac sodium through rat skin from diclofenac sodium solution and diclofenac sodiumsolid lipid nanoparticles suspension was calculated in terms of mean cumulative amount diffused at each sampling time point during a time of $24 \mathrm{~h}$. diclofenac sodium solution exhibited a greater cumulative amount of drug permeation in $24 \mathrm{~h}$ than diclofenac sodium - solid lipid nanoparticles suspension. The solid lipid nanoparticles suspension possessed a sustained drug release over a period of $24 \mathrm{~h}$. This is because most of the drug from diclofenac sodium solution had penetrated through the rat skin after $24 \mathrm{~h}$, the flux values were calculated only according to the data of the first $8 \mathrm{~h}$. The flux values after $8 \mathrm{~h}$ from solid lipid nanoparticle suspension were found to be $22.23 \pm 3.62$ and $55.46 \pm 3.04 \mathrm{~m} \mathrm{~g} / \mathrm{cm} 2 \mathrm{~h}$ from diclofenac sodium solution. A higher value of flux was obtained for diclofenac sodium solution, which can be due to the absence of the release procedure in the system. The flux value for the suspension was found to be lower, which can be attributed to the slow permeation of diclofenac sodium from the solid lipid nanoparticles owing to the encapsulation of the diclofenac sodium in the lipid core. This phenomenon for diclofenac sodium solution can be attributed to the absence of the release procedure of diclofenac sodium. The solid lipid nanoparticles suspension permeated through rat skin was also without lag time, and this can be ascribed to the presence of surfactants in the system, which can improve the transdermal absorption of drugs [Dongfei, Yifan, Yue, Yubing, Qing, Rui. and Qunwei et al 2010].
The deposition potential of the suspension was assessed at the end of $24 \mathrm{~h}$ after application. Diclofenac sodium percentage remained, percentage deposited, and percentage permeated on the skin was calculated. Highest permeation was obtained for diclofenac sodium solution, which can be attributed to its high flux value. Solid lipid nanoparticles suspension showed higher deposition potential for the diclofenac sodium as compared to the diclofenac sodium solution. The deposition potential of solid lipid nanoparticles was found to be approximately twofold more than the diclofenac sodium solution. Solid lipid nanoparticles with small diameters are propitious to enhance the penetration of nanoparticles into the skin and the controlled release of solid lipid nanoparticles may induce the increase of drug accumulation. These observations confirmed the earlier findings of nanoparticles being deposited in the skin, thus acting as a depot to give sustained release. While getting transported across the skin, solid lipid nanoparticles probably expel diclofenac sodium from the lipid matrix as a consequence of polymorphic transitions occurring in the solid lipid.

\section{CONCLUSION}

In conclusion, nanotechnology has developed recently but getting it into the field of transdermal drug delivery had great effects in many aspects and shapes. The nanoparticles whether made from polymers, semiconductors, or even lipids had prodigious outcomes that will revolve everything in the transdermal drug delivery forever.

The polymer-based nanoparticles suggested the advantages of being stable controlled and sustained drug release. Additionally, functionalization and adjustment of their physicochemical properties, storing over months, and encapsulation of both kinds of molecules hydrophobic and hydrophilic. They are manufactured from biodegradable polymers that can natural like gelatin, chitosan, and dextran and its derivatives. Otherwise, it can be created from synthetic polymers like PLGA, PLA, PCL, and smart polymers.

Although, hollow copper sulfide nanoparticles-mediated transdermal delivery is a technique built on the different optical properties of the nanoparticles that have strong absorbance in the near-infrared region and then have photothermal conversion effects. The applied nanosecond-pulsed near-infrared lased provided very short periods but tremendously high temperature in local regions; in summary what is known as thermal ablation. This technique success enables sustained and controlled transdermal delivery of both macromolecules and macromolecules avoiding the pain and inconvenience of longterm stratum corneum injections and low oral bioavailability.

Moreover, the solid lipid nanoparticles had other promising properties too, to deliver diclofenac sodium transdermally into the human body. The drug release from the solid lipid nanoparticles appeared to have two components with a slower exponential release and immediate release. The slower exponential release maintained stable concentrations for longer periods. Solid lipid nanoparticles had been shown to improve the dermal localization of diclofenac sodium, which is one 


\section{International Journal of Engineering Applied Sciences and Technology, 2020 \\ Vol. 5, Issue 2, ISSN No. 2455-2143, Pages 27-36 \\ Published Online June 2020 in IJEAST (http://www.ijeast.com)}

reason to use solid lipid nanoparticles for transdermal drug delivery.

In the end, it is very important to know the latest updates and to compare different ways and solutions to the same challenge in order to make a new powerful one, and that is why this review is made.

\section{ACKNOWLEDGEMENTS}

Dr. Samar Youssef is thanked and acknowledged for her help through the revising of the paper review and her precious advices in editing it.

\section{REFERENCE}

[1] Alkilani, A. Z., McCrudden, M. T., and Donnelly, R. F. (2015). Transdermal Drug Delivery: Innovative Pharmaceutical Developments Based on Disruption of the Barrier Properties of the stratum corneum, (pp.438-470).

[2] Anderson, S. E., Umbright, C., Sellamuthu, R., Fluharty, K., Kashon, M., Franko, J., Jackson, L. G., Johnson, V. J., and Joseph, P. (2010). Irritancy and allergic responses induced by topical application of orthophthalaldehyde. (pp.435-443) .

[3] Anselmo, A. C., and Mitragotri, S. (2019). Nanoparticles in the clinic: An update. (pp.50-60)

[4] Badri W., Eddabra R., Fessi H. and Elaissari A. (2014). Biodegradable Polymer Based Nanoparticles: Dermal and Transdermal Drug Delivery. (pp.141-149).

[5] Baraf H., Fuentealba C., Greenwald M., Brzezicki J., O’Brien K., Soffer B., Polis A., Bird S., Kaur A., Curtis S. and EDGE Study Group (2007). Gastrointestinal side effects of etoricoxib in patients with osteoarthritis: Results of the etoricoxib versus diclofenac sodium gastrointestinal tolerability and effectiveness Trial. (pp.20-488).

[6] Barenholz Y. (2012). Doxil@--the first FDA-approved nano-drug: lessons learned. (pp.117-134).

[7] Cevc G., Vierl U. (2010) Nanotechnology and the transdermal route: A state of the art review and critical appraisal. (pp.99-277).

[8] Chen, J., Li, X., Zhao, X., Wu, Q., Zhu, H., Mao, Z., and Gao, C. (2018). Doxorubicin-conjugated pH-responsive gold nanorods for combined photothermal therapy and chemotherapy of cancer. (pp.347-354).

[9] de Jesus, M. B., and Zuhorn, I. S. (2015). Solid lipid nanoparticles as nucleic acid delivery system: properties and molecular mechanisms. (pp.1-13).

[10]De Jong, W. H., and Borm, P. J. (2008). Drug delivery and nanoparticles:applications and hazards. (pp.133-149).

[11]Dickinson E. (2009) "Hydrocolloids as emulsifiers and emulsion stabilizers.” (pp.253-290)

[12]Dickerson, E. B., Dreaden, E. C., Huang, X., El-Sayed, I. H., Chu, H., Pushpanketh, S., McDonald, J. F., and ElSayed, M. A. (2008). Gold nanorod assisted near-infrared plasmonic photothermal therapy (PPTT) of squamous cell carcinoma in mice. (pp.57-66).
[13]Din, F. U., Aman, W., Ullah, I., Qureshi, O. S., Mustapha, O., Shafique, S., and Zeb, A. (2017). Effective use of nanocarriers as drug delivery systems for the treatment of selected tumors. pp.7291-7309).

[14] Dongfei L., Yifan G., Yue T., Yubing Y., Qing Z., Rui L. and Qunwei X. (2010) Solid lipid nanoparticles for transdermal delivery of diclofenac sodium: Preparation, characterization and in vitro studies. (pp.523-566)

[15]Ezzati Nazhad Dolatabadi, J., Valizadeh, H., and Hamishehkar, H. (2015). Solid Lipid Nanoparticles as Efficient Drug and Gene Delivery Systems: Recent Breakthroughs. (pp. 151-159).

[16]Farjadian, F., Ghasemi, A., Gohari, O., Roointan, A., Karimi, M., and Hamblin, M. R. (2019). Nanopharmaceuticals and nanomedicines currently on the market: challenges and opportunities. (pp.93-126).

[17]Feng, Q., Zhang, Y., Zhang, W., Shan, X., Yuan, Y., Zhang, H., Hou, L., and Zhang, Z. (2016). Tumor-targeted and multi-stimuli responsive drug delivery system for nearinfrared light induced chemo-phototherapy and photoacoustic tomography. (pp.129-142).

[18] Glenn, G. M., Flyer, D. C., Ellingsworth, L. R., Frech, S. A., Frerichs, D. M., Seid, R. C., and Yu, J. (2007). Transcutaneous immunization with heat-labile enterotoxin: development of a needle-free vaccine patch. (pp.809-819).

[19] Glenn, G. M., and Kenney, R. T. (2006). Mass vaccination: solutions in the skin. (pp.247-268).

[20] Godin B., Touitou E. (2009) Transdermal skin delivery: Predictions for humans from in vivo, ex vivo and animal models. (pp.154-164).

[21] Haeri, A., Sadeghian, S., Rabbani, S., Anvari, M. S., Ghassemi, S., Radfar, F., and Dadashzadeh, S. (2017). (pp.1461-1469).

[22]Hu, M., Chen, J., Li, Z. Y., Au, L., Hartland, G. V., Li, X., Marquez, M., and Xia, Y. (2006). Gold nanostructures: engineering their plasmonic properties for biomedical applications. (pp.1084-1094).

[23] Jahan, S. T., Sadat, S., Walliser, M., and Haddadi, A. (2017). Targeted Therapeutic Nanoparticles: An Immense Promise to Fight against Cancer.(pp.755-786)

[24] Jain, P. K., Lee, K. S., El-Sayed, I. H., and El-Sayed, M. A. (2006). Calculated absorption and scattering properties of gold nanoparticles of different size, shape, and composition: applications in biological imaging and biomedicine. (pp.7238-7248).

[25] Jeevanandam, J., Barhoum, A., Chan, Y. S., Dufresne, A., and Danquah, M. K. (2018). Review on nanoparticles and nanostructured materials: history, sources, toxicity and regulations. (1050-1074).

[26] Joshi M., Patravale V. (2008) Nanostructured lipid carrier (NLC) based gel of celecoxib. (pp.124-138).

[27] Kim, H., Beack, S., Han, S., Shin, M., Lee, T., Park, Y., Kim, K. S., Yetisen, A. K., Yun, S. H., Kwon, W., and Hahn, S. K. (2018). Multifunctional Photonic 


\section{International Journal of Engineering Applied Sciences and Technology, 2020 \\ Vol. 5, Issue 2, ISSN No. 2455-2143, Pages 27-36 \\ Published Online June 2020 in IJEAST (http://www.ijeast.com)}

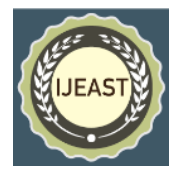

Nanomaterials for Diagnostic, Therapeutic, and Theranostic Applications. (pp.50-75)

[28] Kulkarni, A. D., Vanjari, Y. H., Sancheti, K. H., Belgamwar, V. S., Surana, S. J., and Pardeshi, C. V. (2015). Nanotechnology-mediated nose to brain drug delivery for Parkinson's disease: a mini review. (pp.775788).

[29] Kuo, T. R., Hovhannisyan, V. A., Chao, Y. C., Chao, S. L., Chiang, S. J., Lin, S. J., Dong, C. Y., and Chen, C. C. (2010). Multiple release kinetics of targeted drug from gold nanorod embedded polyelectrolyte conjugates induced by near-infrared laser irradiation. (pp.14163-14171).

[30] Laserra, S., Basit, A., Sozio, P., Marinelli, L., Fornasari, E., Cacciatore, I., Ciulla, M., Türkez, H., Geyikoglu, F., and Di Stefano, A. (2015). Solid lipid nanoparticles loaded with lipoyl-memantine codrug: preparation and characterization. (pp.183-191).

[31]Lee, J. W., Gadiraju, P., Park, J. H., Allen, M. G., and Prausnitz, M. R. (2011). Microsecond thermal ablation of skin for transdermal drug delivery. (pp.58-68).

[32]Li, Y., Lu, W., Huang, Q., Huang, M., Li, C., and Chen, W. (2010). Copper sulfide nanoparticles for photothermal ablation of tumor cells. (pp.1161-1171).

[33] Madamsetty, V. S., , Pal, K., , Keshavan, S., , Caulfield, T. R., , Dutta, S. K., , Wang, E., , Fadeel, B., , and Mukhopadhyay, D., (2019). Development of multi-drug loaded PEGylated nanodiamonds to inhibit tumor growth and metastasis in genetically engineered mouse models of pancreatic cancer. (pp.22006-22018).

[34] Matea, C. T., Mocan, T., Tabaran, F., Pop, T., Mosteanu, O., Puia, C., Iancu, C., and Mocan, L. (2017). Quantum dots in imaging, drug delivery and sensor applications. (pp.5421-5431).

[35] Melancon, M. P., Zhou, M., and Li, C. (2011). Cancer theranostics with near-infrared light-activatable multimodal nanoparticles. (pp.947-956).

[36] Murthy S. K. (2007). Nanoparticles in modern medicine: state of the art and future challenges. (pp.129-141).

[37] Myers R., Oldham K. and Tocci S. (2006) "Holt Chemistry" United States of America, Holt, Rinehart and Winston. (pp.676-704)

[38] Ogura, M., Paliwal, S., and Mitragotri, S. (2008). Lowfrequency sonophoresis: current status and future prospects. (pp.1218-1223).

[39] Palmer B., DeLouise L. (2016) "Nanoparticle-Enabled Transdermal Drug Delivery Systems for Enhanced Dose Control and Tissue Targeting".

[40] Park, J. H., Lee, J. W., Kim, Y. C., and Prausnitz, M. R. (2008). The effect of heat on skin permeability. (pp.94103).

[41]Park, J. S., Yi, S. W., Kim, H. J., Kim, S. M., and Park, K. H. (2016). Regulation of Cell Signaling Factors Using PLGA Nanoparticles Coated/Loaded with Genes and Proteins for Osteogenesis of Human Mesenchymal Stem Cells. ACS (pp.30387-30397)
[42] Pitsillides, C. M., Joe, E. K., Wei, X., Anderson, R. R., and Lin, C. P. (2003). Selective cell targeting with lightabsorbing microparticles and nanoparticles. (pp.40234032).

[43] Prausnitz, M. R., Mitragotri, S., and Langer, R. (2004). Current status and future potential of transdermal drug delivery. (pp.115-124).

[44] Prausnitz, M. R., and Langer, R. (2008). Transdermal drug delivery. (pp.1261-1268).

[45] Puglia, C., Offerta, A., Carbone, C., Bonina, F., Pignatello, R., and Puglisi, G. (2015). Lipid nanocarriers (LNC) and their applications in ocular drug delivery. (pp.1589-1602).

[46] Ramadan S., Guo L., Li Y., YanB. and Lu W. (2012) Hollow Copper Sulfide Nanoparticle-Mediated Transdermal Drug Delivery NIH Public Access.

[47] Rostami, E., Kashanian, S., Azandaryani, A. H., Faramarzi, H., Dolatabadi, J. E., and Omidfar, K. (2014). Drug targeting using solid lipid nanoparticles. (pp.56-61).

[48] Schoellhammera C., Blankschtein D. and Langer R. (2014) Skin Permeabilization for Transdermal Drug Delivery: Recent Advances and Future Prospects.NIH Public Access.

[49] Schroeter A, Engelbrecht T., Neubert R., and Goebel A. (2010) New Nanosized Technologies for Dermal and Transdermal Drug Delivery. A Review. (pp.511-528).

[50] Sharma, A., Garg, T., Aman, A., Panchal, K., Sharma, R., Kumar, S., and Markandeywar, T. (2016). Nanogel--an advanced drug delivery tool: Current and future. (pp.165177).

[51] Shreffler, J. W., Pullan, J. E., Dailey, K. M., Mallik, S., and Brooks, A. E. (2019). Overcoming Hurdles in Nanoparticle Clinical Translation: The Influence of Experimental Design and Surface Modification. (pp.351-380)

[52] Skrabalak, S. E., Chen, J., Au, L., Lu, X., Li, X., and Xia, Y. (2007). Gold Nanocages for Biomedical Applications.

[53] Song, S., Xiong, C., Zhou, M., Lu, W., Huang, Q., Ku, G., Zhao, J., Flores, L. G., Jr, Ni, Y., and Li, C. (2011). Smallanimal PET of tumor damage induced by photothermal ablation with 64Cu-bis-DOTA-hypericin. (pp.792-799).

[54] Suzuki, T., Miyazaki, C., Ishii-Watabe, A., Tada, M., Sakai-Kato, K., Kawanishi, T., and Kawasaki, N. (2015). A fluorescent imaging method for analyzing the biodistribution of therapeutic monoclonal antibodies that can distinguish intact antibodies from their breakdown products. (pp.759-769).

[55] Wiedersberg S., Guy R. (2014) “Transdermal Drug Delivery: 30+ Years of War and Still Fighting!" (pp.150156).

[56] Wolfram, J., Zhu, M., Yang, Y., Shen, J., Gentile, E., Paolino, D., Fresta, M., Nie, G., Chen, C., Shen, H., Ferrari, M., and Zhao, Y. (2015). Safety of Nanoparticles in Medicine. (pp.1671-1681).

[57] Wu, G., Mikhailovsky, A., Khant, H. A., Fu, C., Chiu, W., and Zasadzinski, J. A. (2008). Remotely triggered liposome release by near-infrared light absorption via hollow gold nanoshells. (pp.8175-8177) 
[58] You, J., Zhang, G., and Li, C. (2010). Exceptionally high payload of doxorubicin in hollow gold nanospheres for near-infrared light-triggered drug release. (pp.1033-1041).

[59] Yu, J., Kalaria, D. R., and Kalia, Y. N. (2011). Erbium:YAG fractional laser ablation for the percutaneous delivery of intact functional therapeutic antibodies. (pp.53-59).

[60]Zhou, M., Zhang, R., Huang, M., Lu, W., Song, S., Melancon, M. P., Tian, M., Liang, D., and Li, C. (2010). A chelator-free multifunctional $[64 \mathrm{Cu}] \mathrm{CuS}$ nanoparticle platform for simultaneous micro-PET/CT imaging and photothermal ablation therapy.

[61]Zhu, H., Wang, J., and Wu, D. (2009). Fast synthesis, formation mechanism, and control of shell thickness of CuS hollow spheres. (pp.7099-7104). 